\title{
Efficacy of cytokine-induced killer cells targeting CD40 and GITR
}

\author{
KAJA D. BIEDERBICK ${ }^{1}$ and INGO G.H. SCHMIDT-WOLF ${ }^{2}$ \\ ${ }^{1}$ Department of Internal Medicine III, University Hospital Bonn; ${ }^{2}$ Department of Integrated \\ Oncology, CIO Bonn, University Hospital Bonn, D-53105 Bonn, Germany
}

Received October 30, 2017; Accepted September 25, 2018

DOI: $10.3892 / \mathrm{ol} .2018 .9849$

\begin{abstract}
Since the publication of a novel protocol in 1991, cytokine-induced killer (CIK) cells have shown promising results in the treatment against neoplastic diseases. Despite ongoing preclinical and clinical studies, CIK cell treatment in the context of human monoclonal antibodies targeting tumor-necrosis factor receptors remains overlooked. The present study investigated whether a combination of CIK cells with human monoclonal antibody anti-CD40 and anti-Glucocorticoid-induced TNF-related protein (GITR) would lead to further cytotoxicity against tumor cells expressing CD40 and GITR ligand (L). Therefore, in vitro experiments with human lymphoma cell lines SU-DHL-4 and Daudi (both CD40 positive) and human breast adenocarcinoma MCF-7 (GITRL positive) were performed and the secretion of interferon (IFN) $\gamma$ was measured. Three interesting results emerged: i) a combination of CIK cells and anti-CD40 mAb is more effective than CIK cell treatment alone; ii) the use of anti-GITR mAb and CIK cells significantly enhanced the cytotoxicity of CIK cells against MCF-7 compared with single CIK cell treatment and iii) the combination of both antibodies and CIK cells abrogates the anti tumoral effect of CIK cells on all three cell lines. By performing an ELISA for IFN- $\gamma$ measurement, a lower secretion was observed when anti-CD40 or anti-GITR mAb was added. This outcome indicates that further studies in vitro and in vivo may aid in understanding the synergistic molecular mechanisms of CIK cells, and anti-CD40 and anti-GITR mAb.
\end{abstract}

Correspondence to: Professor Ingo G.H. Schmidt-Wolf, Department of Integrated Oncology, CIO Bonn, University Hospital Bonn, 25 Sigmund-Freud-Straße, D-53105 Bonn, Germany

E-mail: ingo.schmidt-wolf@ukbonn.de

Abbreviations: GITRL, Glucocorticoid-induced TNF-related protein ligand; $\mathrm{TNF}$, tumor necrosis factor, NF- $\kappa \mathrm{B}$, nuclear factor- $\mathrm{\kappa B}$; IL, interleukin, MHC, major histocompatibility complex; NKG2D, natural killer group 2, member D

Key words: cytokine-induced killer cells, monoclonal antibody, anti-CD40, anti-GITR, immunotherapy

\section{Introduction}

There are certain cell surface proteins which are expressed on tumor cells and help them to suppress the immune system, grow and disperse in the human body. In this study, we tested whether this interaction could be blocked by the addition of human monoclonal antibodies targeting members of the tumor necrosis factor receptor (TNFR) superfamily. Two of these members are CD40 and glucocorticoid-induced TNF-related protein (GITR). Both of them use a cascade which requires TNFR-associated factors (TRAFs). The binding of TNFR to TRAFs initiates different signal transductions which lead among other things to the upregulation of NF- $\kappa B(1)$.

CD40 is normally found on antigen-presenting cells and B-cells and plays an important role in primary immune response, B-cell activation and antibody-dependent cell cytotoxity. However, it is also expressed on various hematological malignancies such as multiple myeloma $(2,3)$, non-Hodgkin's lymphoma (4) and Hodgkin's disease (5). The ligand (L) for CD40 is also a TNF superfamily member (CD154) $(6,7)$ and can be found on activated CD4+, CD8+ and $\gamma \delta$ T cells $(6,8)$. On malignant B cells, signaling via CD40 can lead to proapoptotic or antiapoptotic effects on tumor cells (9).

GITR can be found on CD4+ and CD8+ T cells as well as on natural killer (NK) cells and is highly expressed after activation (10). Studies have shown that GITRL-positive tumors such as MCF-7 use the GITR-GITRL-interaction to avoid immune response by the secretion of TNF- $\alpha$, IL- 6 and IL-8 (11,12).

Cytokine-induced killer (CIK) cells are a heterogenous cell group consisting of CD3+CD56+, CD3-CD56+ and CD3+CD56- $T$ cells which exercise their cytotoxicity in a non MHC-restricted manner (13). The $\mathrm{CD}^{+} \mathrm{CD}^{2} 6^{+}$subpopulation serves as the main effector cells combining $\mathrm{T}$ cell capability with NK cell function. Tumor lysis is finalized via NKG2D receptor and MHC-related ligands (MIC A/B) and ULBP family on tumor cells which results in up-regulated secretion of perforin and granzym (14-16). CIK cells have already been proven to be a promising treatment against malignant diseases in various preclinical and clinical studies (17-24). Recent developments in immunotherapy show interesting outcomes and our aim in this study was to find new ways to make CIK cell treatment even more effective by combining them with monoclonal antibodies which are supposed to block tumor surviving interaction between malignant cells and CIK cells. 


\section{Materials and methods}

Antibodies. Human monoclonal antibody anti-GITR (clone 110416) and anti-CD40 (clone 82102) were obtained from R\&D Systems Europe, Ltd., (Abingdon, UK) and dissolved in sterile phosphate buffered saline (PBS) to a concentration of 10 and $1 \mu \mathrm{g} / \mathrm{mL}$ for experimental use. Mouse IgG1 isotyope control and mouse IgG2B isotype control (R\&D Systems) were kept in sterile PBS in a concentration of 10 and $1 \mu \mathrm{g} / \mathrm{mL}$.

Tumor cells. The human breast adenocarcinoma cell line MCF-7 (DSMZ, Braunschweig, Germany) was cultured in DMEM medium (Thermo Fisher Scientific, Inc., Waltham, MA, USA) supplemented with $10 \%$ fetal calf serum (FCS) and $1 \%$ penicillin/streptomycin (P/S) (both from Invitrogen; Thermo Fisher Scientific, Inc.) while the human lymphoma cells SU-DHL-4 and Daudi (DSMZ) were kept in RPMI-1640 medium (Pan-Biotech $\mathrm{GmbH}$, Aidenbach, Germany) containing 10\% FCS and 1\% penicillin/streptomycin. All three cell lines were incubated at $37^{\circ} \mathrm{C}$ in a humidified atmosphere of $5 \% \mathrm{CO}_{2}$.

Generation of CIK cells. CIK cells were generated as described previously (25). Peripheral mononuclear blood lymphocytes were derived from healthy donors. After having washed cells for the first time with PBS $+10 \%$ bovine serum albumin (BSA), the cell pellet was resuspended in $10 \mathrm{ml}$ erythrocyte lysis puffer. Cells were then washed twice again, counted and cultured in Hepes buffered RPMI-1640 medium complemented with $10 \%$ FCS and $1 \% \mathrm{P} / \mathrm{S}$ at a density of $5 \times 10^{\wedge} 6 / \mathrm{ml}$. To separate CIK cells from dendritic cells, the non-adherent cells containing medium were transferred into a new bottle after $1-2 \mathrm{~h}$ of incubation at $37^{\circ} \mathrm{C}$. Medium was filled up to a total volume of $40 \mathrm{ml}$ and 1,000 U/ml recombinant human interferon gamma (IFN-y) (ImmunoTools GmbH, Friesoythe, Germany) was added. On day one, CIK cell received 1,000 U/ml anti-CD3 (eBioscience; Thermo Fisher Scientific, Inc.), 1,000 U/ml IL-1ß (ImmunoTools $\mathrm{GmbH}$ ) and $300 \mathrm{U} / \mathrm{ml}$ rh IL-2 (ImmunoTools $\mathrm{GmbH}$ ). Every third day, medium was partially changed and additional rh IL-2 (300 U/ml) was given. After 2 weeks of maturation, CIK cells were ready for experimental use.

MTT assay. In order to determine CIK cell cytotoxicity, an MTT assay was performed. Therefore, tumor cells $\left(1 \times 10^{\wedge} 4\right.$ cells/well $)$ were seeded in triplicates into round-bottomed 96 well plates. Human monoclonal antibody or isotype control and CIK cells at an effector-to-target ratio of 1:5 were added to a total volume of $100 \mu \mathrm{l} /$ well and the plates were incubated $24 \mathrm{~h}$ at $37^{\circ} \mathrm{C}$. After that, $80 \mu \mathrm{l}$ of the supernatant was removed and $55 \mu 1$ MTT-reagent 3-(4,5-dimethylthiazol-2-yl)-2,5diphenyltetrazolium bromide was given to the cells. Vital cells were able to include the yellow colored reagent and reduce it to purple formazan. Cell viability depended on the amount of measured dye. After $45 \mathrm{~min}$ of incubation, the MTT colorant was dislodged and cells were lysed with DMSO (Carl Roth GmbH, Co., KG, Karlsruhe, Germany). The plate was shaken for ten min at $300 \mathrm{rpm}$ and absorbance was measured at $560 \mathrm{~nm}$.

The following formula was used to calculate cell viability: Viability $(\%)=100-[($ tumor cells incubated with CIK cells with or without additional antibogy -CIK only)/tumor only] $\mathrm{x} 100$
This experiment was replicated at least three times with CIK cells from different donors.

Determination of cytokines. For IFN-y determination, an ELISA was done using Thermo Scientific Human IFN-y ELISA Reagent Kit (Thermo Fisher Scientific, Inc.). Tumor cells $\left(1 \times 10^{\wedge} 5\right)$ were seeded in quadruplets and incubated with anti-GITR or anti-CD40 $\mathrm{mAb}(10 \mu \mathrm{g} / \mathrm{mL})$ and CIK cells (E:T 1:2) in round bottomed 96-wells plates for $24 \mathrm{~h}$ at $37^{\circ} \mathrm{C}$. This was followed by transferring supernatant $(100 \mu \mathrm{l})$ into 96-wells ELISA plates, precoated with IFN-y coating antibody and following the instructor's protocol IFN-y concentration, was measured at $450 \mathrm{~nm}$ wavelength.

Statistical analysis. For statistical analysis, GraphPad Prism v5 was used (GraphPad Software, Inc., La Jolla, CA, USA). One-way analysis of variance with Bonferroni post hoc test and Student's t-test were performed to analyze statistical significance. $\mathrm{P}<0.05$ was considered to indicate a statistically significant difference.

\section{Results}

Effect of combining CIK cells with human monoclonal antibody anti-CD4O on lymphoma cell line SU-DHL-4 and Daudi. CIK cells only had a slightly cytolytic effect against SU-DHL-4 and Daudi when cultured alone at a very low effector-to-target ratio (1:5). By adding human monoclonal antibody anti-CD40 $(1 \mu \mathrm{g} / \mathrm{mL})$ the cytotoxicity of CIK cells could be increased by up to $27.19 \%$ for SU-DHL-4 (Fig. 1A) and $28.26 \%$ for Daudi (Fig. 1B). Anti-CD40 itself had no effect on the tumor cell lines. The MTT-assay was also performed using isotype control in the same concentration as anti-CD40 $(1 \mu \mathrm{g} / \mathrm{mL})$. No significant changes were measured (data not shown).

Effect of combining CIK cells with human monoclonal antibody anti-GITR on breast adenocarcinoma cells MCF-7. There was an improvement of cytolysis against MCF-7 when CIK cells were stimulated with anti-GITR mAb $(10 \mu \mathrm{g} / \mathrm{mL})$ compared to single CIK cell treatment (11.38\%) (Fig. 1C). Again, we used a low E:T ratio (1:5) and for controls performed the assay under same circumstances with isotype control. No significant effect was noticed (data not shown). Co-culturing of MCF-7 and anti-GITR mAb had no effect on cell viability.

Detection of IFN- $\gamma$ secretion on single CIK cell treatment and CIK cell/human monoclonal antibody treatment. To investigate further, we measured the IFN- $\gamma$ secretion of CIK cells when cultured with tumor cell lines (SU-HDL-4, Daudi and MCF-7) alone (E:T 1:2) and in the presence of human monoclonal antibody anti-CD40, anti-GITR or isotype control at a concentration of $10 \mu \mathrm{g} / \mathrm{mL}$ (Fig. 2). After $24 \mathrm{~h}$ of incubation the supernatants were analyzed by ELISA. Surprisingly, the amount of IFN- $\gamma$ decreases or remains the same when anti-CD40 or anti-GITR mAb is added. The use of isotype control did not lead to significant changes either (data not shown).

Combination of both antibodies anti-CD4O and anti-GITR with CIK cells on all three tumor cell lines and their effects. 

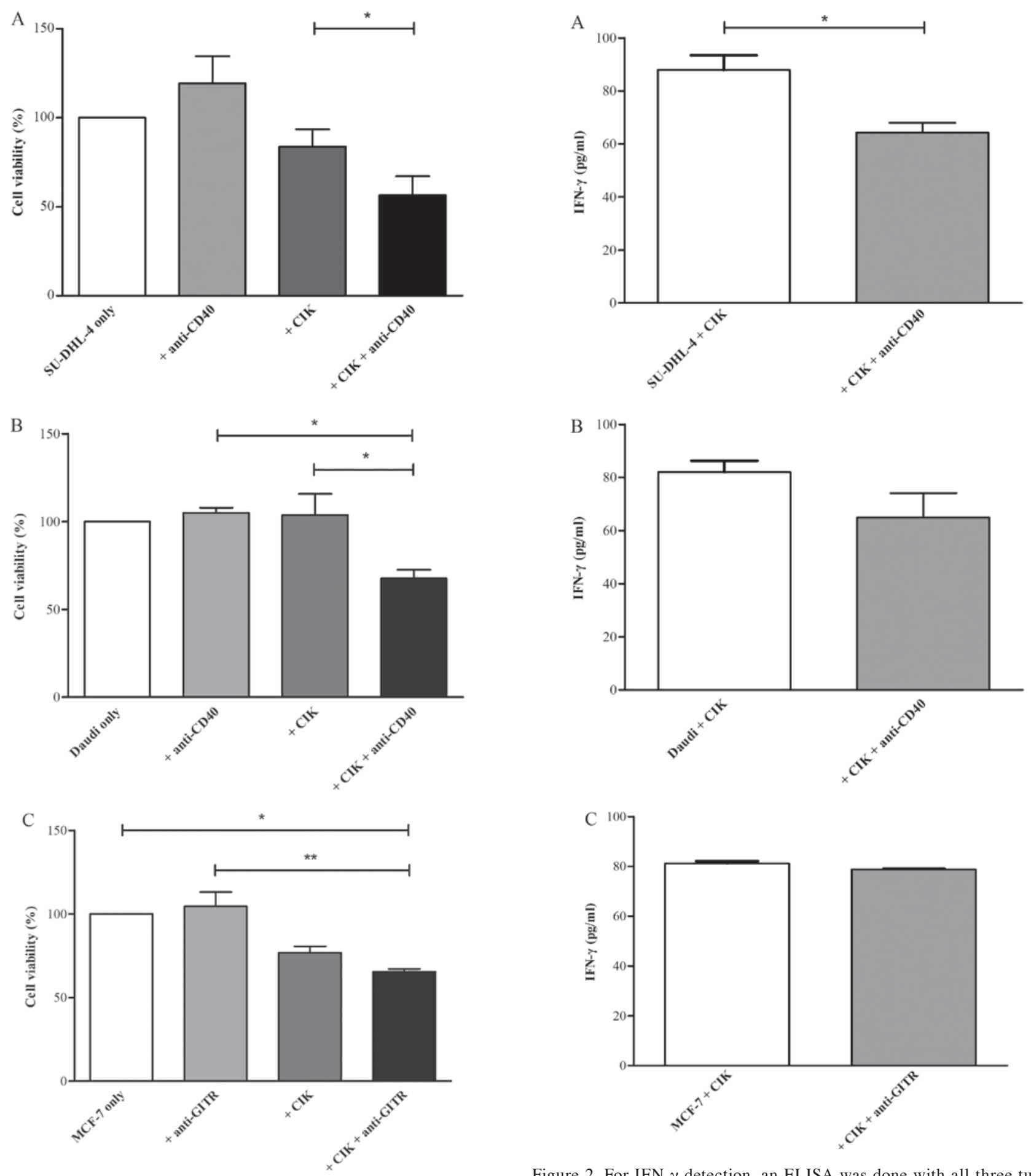

Figure 1. Cell viability was measured using MTT assay. (A) SU-DHL-4 with anti-CD40 cytotoxicity increased up to $27.19 \%(\mathrm{P}=0.0346)$. (B) Daudi with anti-CD40 led to $28.26 \%$ higher cytotoxicity ( $\mathrm{P}=0.0357)$. (C) MCF-7 with anti-GITR improvement of cytolysis of $11.38 \%$ ( $\mathrm{P}=0.0372$ ). Data presented as mean $+/$ - SEM. ${ }^{*} \mathrm{P}<0.05$ and ${ }^{* *} \mathrm{P}<0.01$. anti-GIRT, anti-Glucocorticoid-induced TNF-related protein.

We proceeded to test whether combining the two antibodies would lead to further stimulation of CIK cells. As Fig. 3 demonstrates this process has indeed, the opposite effect. By applying anti-GITR $(10 \mu \mathrm{g} / \mathrm{mL})$ and anti-CD40 $\mathrm{mAb}$ $(1 \mu \mathrm{g} / \mathrm{mL})$ with CIK cells on SU-DHL-4, Daudi and MCF-7, the cytolytic activity of CIK cells appeared to be decreasing on all three tumor cell lines.

\section{Discussion}

Immunomodulatory monoclonal antibodies in the treatment of malignant diseases have gained much interest over the past years. The concept is that monoclonal antibodies block the interaction between certain surface proteins on tumor cells 

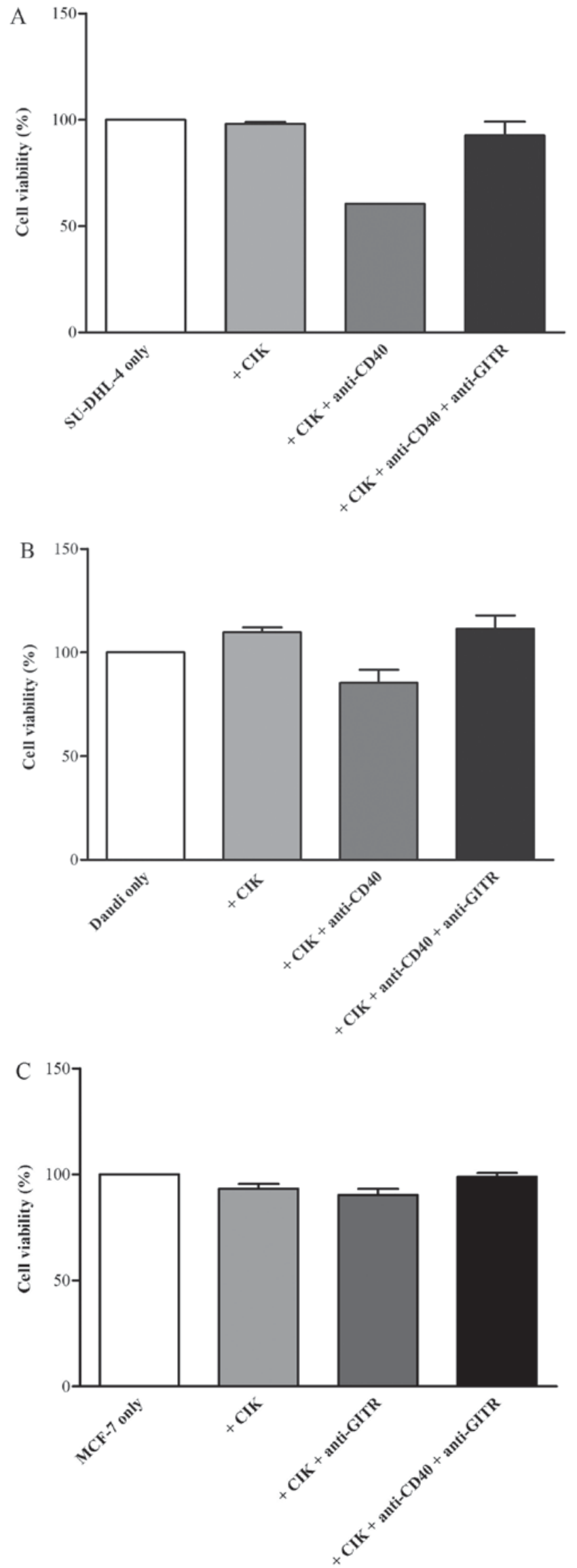

Figure 3. Tumor cell lines (A) SU-DHL-4, (B) Daudi and (C) MCF-7 were co-cultured with CIK alone, CIK cells and anti-CD40 or anti-GITR and with CIK cells and both antibodies. By applying the second antibody the cytolytic activity of CIK cells seems to be inhibited in all three samples. CIK, cytokine-induced killer. and the immune system which normally help them to suppress immune response. Furthermore, human monoclonal antibodies might be able to induce antibody-dependent cell-mediated cytotoxicity (ADCC). It has already been reported that $\mathrm{CD}^{+}{ }^{+} \mathrm{CD} 56^{+}$cells express $\mathrm{CD} 16$ and therefore ADCC might be a possible mechanism on how CIK cells perform their enhanced cytolytic activity in our work (26). Since monoclonal antibodies such as rituximab and ipilimumab have already been approved as therapeutic reagents against few malignant diseases new approaches targeting other molecules than CD20 and CTLA-4 have now become the focus of study. A variety of hematological and non-hematological neoplasias express CD40, so monoclonal antibodies against CD40 might be a promising treatment option for these cell lines. Normally found on B cells, signaling via CD40 can be pro- or antiapoptotic on B-lineage malignancies $(9,27)$. The intracellular pathway still needs to be explored. One explanation might be the induction of bax after CD40-stimulation (28). As part of the Bcl-2 family it facilitates p53 induced apoptosis (29). Humanized agonistic SGN-40 and antagonistic CHIR-12.12 have already been generated and are currently used in clinical trials (27). In our study, we showed that a combination of human monoclonal anti-CD40 with CIK cells led to increased cytotoxicity compared to CIK cell treatment alone against CD40+ lymphoma cells SU-DHL-4 and Daudi. Anti-CD40 $\mathrm{mAb}$ detects the corresponding surface protein with its Fab-fragment on SU-DHL-4 and Daudi while the Fc-region functions as stimulatory signal for CIK cells. Moreover, CD40 is also expressed on CD8+ T cells and following a cascade of Ras, Phosphoinositide 3-kinase (PI3K) and protein kinase $\mathrm{C}$ (PKC) CD40, -signaling results in down regulating T reg cells' immunosuppressive effects (30). In effect, human anti-CD40 $\mathrm{mAb}$ may work in one or both ways to induce anti tumor activity; the exact molecular mechanisms still remain unclear and need to be investigated further.

We tested a second combination of CIK cells with another monoclonal antibody targeting Glucocorticoid-induced TNFR (GITR). Here, the cytolytic activity of CIK cells seems to be enhanced by stimulation with human anti-GITR mAb. GITR is found on CD4+-, CD8+-, NK- and Treg cells where as its ligand, GITRL, is constitutively expressed on tumor cell lines like MCF-7 and uses the GITR-GITRL-interaction for immunosurveillance. The Salih group reported that by GITR-stimulation the $\mathrm{NF}-\kappa \mathrm{B}$ activity in $\mathrm{NK}$ cells was decreased and could be partially regained after addition of anti-GITR. This data indicates that GITR-induced reduction of NF- $\kappa \mathrm{B}$ may explain how GITRL-expressing tumors escape immune defense (10).

Since we examined a higher cytolytic activity of CIK cells by the addition of anti-CD40 or anti-GITR mAb, we expected a correlating increase in IFN- $\gamma$ production when CIK cells were stimulated with human monoclonal antibodies. However, we found that the opposite was true. With human lymphoma cells SU-DHL-4 and Daudi, a lower secretion of IFN- $\gamma$ was measured and no changeable amounts with MCF-7. Partially, these $-\gamma$ increase when anti-GITR was added could not be explained.

Finally, we tested the cytolytic activity of CIK cells when anti-CD40 and anti-GITR mAb were incubated with all three cell lines. In each sample CIK cells' cytotoxicity was inhibited. 
This data has led us to the conclusion that CD40 and GITR share a common pathway. Both molecules belong to the TNFR superfamily and use TRAF proteins for signal transduction (31-33).

Our results confirm the works of Baltz et al Only the treatment with IL-15 enhanced NK cells' production of IFN- $\gamma$ while untreated NK cells showed minor or no effect (10). Why IL-15 was necessary for IFN. This might be an explanation why the simultaneous use of anti-GITR ant anti-CD40 mAb lowered CIK cells' cytotoxicity in comparison to CIK cell treatment with one monoclonal antibody. To sum up, the combination of CIK cells and human monoclonal antibodies showed promising results in vitro. Our findings confirm the works of Cappuzzello et al who recently reported that CIK cells are capable of ADCC and their cytolytic activity increased when monoclonal antibody was added (26). This opens up a variety of combinations between CIK cells and different monoclonal antibodies such as anti-CD137, anti-CD134 (OX40), anti-CD152 (CTLA-4), anti-PD-1 and anti-PD-L1. Most of them are under current clinical investigation (34). Anti-CD137 and anti-CD30 have already been tested with CIK cells $(35,36)$. Immunotherapy, especially the use of monoclonal antibodies, has the potential to replace the present treatments against cancer since it is a restricted therapy targeted to tumor and carries less side effects, and as such, efforts in this field must continue.

\section{Acknowledgements}

The authors would like to thank Mrs. Sabine Blaum-Feder and Ms. Clara Jäkel (Department of Internal Medicine III, University Hospital Bonn, Bonn, Germany) for their professional help.

\section{Funding}

No funding was received.

\section{Availability of data and materials}

All data used and analyzed during the present study are available from the corresponding author on reasonable request.

\section{Authors' contributions}

KB performed the experiments, analyzed the data and wrote the essay. ISW designed and supervised the study, and corrected the manuscript.

\section{Ethics approval and consent to participate}

Not applicable.

\section{Patient consent for publication}

Not applicable.

\section{Competing interests}

The authors declare no competing interests.

\section{References}

1. Bishop GA: The multifaceted roles of TRAFs in the regulation of B-cell function. Nat Rev Immunol 4: 775-786, 2004.

2. Pellat-Deceunynck C, Bataille R, Robillard N, Harousseau JL, Rapp MJ, Juge-Morineau N, Wijdenes $\mathrm{J}$ and Amiot $\mathrm{M}$ : Expression of CD28 and CD40 in human myeloma cells: A comparative study with normal plasma cells. Blood 84: 2597-2603, 1994.

3. Westendorf JJ, Ahmann GJ, Armitage RJ, Spriggs MK, Lust JA, Greipp PR, Katzmann JA and Jelinek DF: CD40 expression in malignant plasma cells. Role in stimulation of autocrine IL-6 secretion by a human myeloma cell line. J Immunol 152: 117-128, 1994.

4. Uckun FM, Gajl-Peczalska K, Myers DE, Jaszcz W, Haissig S and Ledbetter JA: Temporal association of CD40 antigen expression with discrete stages of human B-cell ontogeny and the efficacy of anti-CD40 immunotoxins against clonogenic B-lineage acute lymphoblastic leukemia as well as B-lineage non-Hodgkin's lymphoma cells. Blood 76: 2449-2456, 1990.

5. O'Grady JT, Stewart S, Lowrey J, Howie SE and Krajewski AS: CD40 expression in Hodgkin's disease. Am J Pathol 144: 21-26, 1994.

6. van Kooten C and Banchereau J: CD40-CD40 ligand. J Leukoc Biol 67: 2-17, 2000.

7. Banchereau J, Bazan F, Blanchard D, Brière F, Galizzi JP, van Kooten C, Liu YJ, Rousset F and Saeland S: The CD40 antigen and its ligand. Annu Rev Immunol 12: 881-922, 1994.

8. Law CL, Gordon KA, Collier J, Klussman K, McEarchern JA, Cerveny CG, Mixan BJ, Lee WP, Lin Z, Valdez P, et al: Preclinical antilymphoma activity of a humanized anti-CD40 monoclonal antibody, SGN-40. Cancer Res 65: 8331-8338, 2005.

9. Costello RT, Gastaut JA and Olive D: What is the real role of CD40 in cancer immunotherapy? Immunol Today 20: 488-493, 1999.

10. Baltz KM, Krusch M, Bringmann A, Brossart P, Mayer F, Kloss M, Baessler T, Kumbier I, Peterfi A, Kupka S, et al: Cancer immunoediting by GITR (glucocorticoid-induced TNF-related protein) ligand in humans: NK cell/tumor cell interactions. FASEB J 21: 2442-2454, 2007.

11. Baessler T, Krusch M, Schmiedel BJ, Kloss M, Baltz KM, Wacker A, Schmetzer HM and Salih HR: Glucocorticoid-induced tumor necrosis factor receptor-related protein ligand subverts immunosurveillance of acute myeloid leukemia in humans. Cancer Res 69: 1037-1045, 2009.

12. Buechele C, Baessler T, Wirths S, Schmohl JU, Schmiedel BJ and Salih HR: Glucocorticoid-induced TNFR-related protein (GITR) ligand modulates cytokine release and NK cell reactivity in chronic lymphocytic leukemia (CLL). Leukemia 26: 991-1000, 2012.

13. Schmidt-Wolf IG, Lefterova P, Mehta BA, Fernandez LP, Huhn D, Blume KG, Weissman IL and Negrin RS: Phenotypic characterization and identification of effector cells involved in tumor cell recognition of cytokine-induced killer cells. Exp Hematol 21: 1673-1679, 1993.

14. Nwangwu CA, Weiher $\mathrm{H}$ and Schmidt-Wolf IGH: Increase of CIK cell efficacy by upregulating cell surface MICA and inhibition of NKG2D ligand shedding in multiple myeloma. Hematol Oncol 35, 2016

15. He JY, Jia ZX, Cai XH, Han WM, Xiao R, Ma LD, Lu XZ,Zhou M and Chen BA: Roles of NKG2D in cytokine-induced killer (CIK) against hematological malignant cells lines. Zhongguo Shi Yan Xue Ye Xue Za Zhi 21: 1380-1384, 2013 (In Chinese).

16. Verneris MR, Karimi M, Baker J, Jayaswal A and Negrin RS: Role of NKG2D signaling in the cytotoxicity of activated and expanded CD8+ T cells. Blood 103: 3065-3072, 2004.

17. Zhao H, Fan Y, Li H, Yu J, Liu L, Cao S, Ren B, Yan F and Ren X: Immunotherapy with cytokine-induced killer cells as an adjuvant treatment for advanced gastric carcinoma: A retrospective study of 165 patients. Cancer Biother Radiopharm 28: 303-309, 2013.

18. Hao MZ, Lin HL, Chen Q, Ye YB, Chen QZ and Chen MS: Efficacy of transcatheter arterial chemoembolization combined with cytokine-induced killer cell therapy on hepatocellular carcinoma: A comparative study. Chin J Cancer 29: 172-177, 2010.

19. Yang B, Lu XC, Yu RL, Chi XH, Liu Y, Wang Y, Dai HR, Zhu HL, Cai LL and Han WD: Repeated transfusions of autologous cytokine-induced killer cells for treatment of haematological malignancies in elderly patients: A pilot clinical trial. Hematol Oncol 30: 115-122, 2012.

20. Schmeel LC, Schmeel FC, Coch C and Schmidt-Wolf IG: Cytokine-induced killer (CIK) cells in cancer immunotherapy: Report of the international registry on CIK cells (IRCC). J Cancer Res Clin Oncol 141: 839-849, 2015. 
21. Wang Y, Xu Z, Zhou F, Sun Y, Chen J, Li L, Jin H and Qian Q: The combination of dendritic cells-cytotoxic T lymphocytes/cytokine-induced killer (DC-CTL/CIK) therapy exerts immune and clinical responses in patients with malignant tumors. Exp Hematol Oncol 4: 32, 2015.

22. Wang Z, Liu Y, Li R, Shang Y, Zhang Y, Zhao L, Li W, Yang Y, Zhang X, Yang T, et al: Autologous cytokine-induced killer cell transfusion increases overall survival in advanced pancreatic cancer. J Hematol Oncol 9: 6, 2016.

23. Jäkel CE and Schmidt-Wolf IG: An update on new adoptive immunotherapy strategies for solid tumors with cytokine-induced killer cells. Expert Opin Biol Ther 14: 905-916, 2014.

24. Jäkel CE, Vogt A, Gonzalez-Carmona MA and Schmidt-Wolf IG: Clinical studies applying cytokine-induced killer cells for the treatment of gastrointestinal tumors. J Immunol Res 2014: 897214, 2014.

25. Schmidt-Wolf IG, Negrin RS, Kiem HP, Blume KG and Weissman IL: Use of a SCID mouse/human lymphoma model to evaluate cytokine-induced killer cells with potent antitumor cell activity. J Exp Med 174: 139-149, 1991.

26. Cappuzzello E, Tosi A, Zanovello P, Sommaggio R and Rosato A Retargeting cytokine-induced killer cell activity by CD16 engagement with clinical-grade antibodies. Oncoimmunology 5 : e1199311, 2016.

27. Hassan SB, Sørensen JF, Olsen BN and Pedersen AE: Anti-CD40-mediated cancer immunotherapy: An update of recent and ongoing clinical trials. Immunopharmacol Immunotoxicol 36: 96-104, 2014.

28. Szocinski JL, Khaled AR, Hixon J, Halverson D, Funakoshi S, Fanslow WC, Boyd A, Taub DD, Durum SK, Siegall CB, et al: Activation-induced cell death of aggressive histology lymphomas by CD40 stimulation: Induction of bax. Blood 100: 217-223, 2002.

29. Brady HJM and Gil-Gómez G: Molecules in focus Bax. The pro-apoptotic Bcl-2 family member, Bax. Int J Bio Cell Biol 30 647-650, 1998
30. Martin S, Pahari S, Sudan R and Saha B: CD40 signaling in $\mathrm{CD} 8+\mathrm{CD} 40+\mathrm{T}$ cells turns on contra-T regulatory cell functions. J Immunol 184: 5510-5518, 2010.

31. McWhirter SM, Pullen SS, Holton JM, Crute JJ, Kehry MR and Alber T: Crystallographic analysis of CD40 recognition and signaling by human TRAF2. Proc Natl Acad Sci USA 96: 8408-8413, 1999.

32. Tsukamoto N, Kobayashi N, Azuma S, Yamamoto T and Inoue J: Two differently regulated nuclear factor kappaB activation pathways triggered by the cytoplasmic tail of CD40. Proc Natl Acad Sci USA 96: 1234-1239, 1999.

33. Esparza EM and Arch RH: Glucocorticoid-induced TNF receptor, a costimulatory receptor on naive and activated $\mathrm{T}$ cells, uses TNF receptor-associated factor 2 in a novel fashion as an inhibitor of NF-kappa B activation. J Immunol 174: 7875-7882, 2005.

34. Melero I, Grimaldi AM, Perez-Gracia JL and Ascierto PA: Clinical development of immunostimulatory monoclonal antibodies and opportunities for combination. Clin Cancer Res 19: 997-1008, 2013.

35. Zhu BQ, Ju SW and Shu YQ: CD137 enhances cytotoxicity of CD3(+)CD56(+) cells and their capacities to induce CD4(+) Th1 responses. Biomed Pharmacother 63: 509-516, 2009.

36. Esser L, Weiher $\mathrm{H}$ and Schmidt-Wolf I: Increased efficacy of brentuximab vedotin (SGN-35) in combination with cytokine-induced killer cells in lymphoma. Int J Mol Sci 17: pii: E1056, 2016. 